\section{Ernährungsberatung: Individuell statt pauschal!}

A nne Kamp, Neuenkirchen, stellte das Krankheitsbild der Histaminunverträglichkeit dar. Sie machte deutlich, dass die Empfindlichkeit gegenüber Histamin abhängig von verschiedenen Einflussfaktoren schwanken kann. Dabei veranschaulichte sie anhand eigener Daten, dass Pauschallisten nicht geeignet sind, die Betroffenen ernährungstherapeutisch zu führen. So werden häufig beschriebene Auslöser wie Banane, Tomate oder Himbeere von vielen Patienten problemlos vertragen. Aber auch für weniger gut verträgliche Nahrungsmittel wie Rotwein oder gereiften Käse lassen sich oft individuelle Toleranzgrenzen ermitteln, so dass eine strenge Karenz nur selten erforderlich ist. Die Meidung von Hefe, wie häufig empfohlen, sei gänzlich unnötig, erklärte Kamp, da der Gehalt an biogenen Aminen unerheblich sei.

Einen neuen Ansatz zur Diagnostik einer Pseudoallergie bei Patienten mit chronischer Urtikaria stellte Britta Bunselmeyer, Münster, vor. Im Gegensatz zur oralen Provokation mit einer pseudoallergenreichen Mahlzeit, wie in den Leitlinien der DGAKI empfohlen, durchliefen Betroffene an der Uniklinik in Münster im Anschluss an eine Besserung unter pseudoallergenarmer Diät eine sechswöchige Aufbaukost. Im Rahmen dieses Programmes wurden mögliche Auslösergruppen (natürliche Aromastoffe, biogene Amine, Zusatzstoffe) auf ihre Relevanz hin ausgetestet. Der Vorteil ihres Ansatzes liege in der individuellen Ausrichtung der Provokationskost, erklärte Bunselmeyer, die Aufbaukost sei weiterhin geeignet, direkt in die therapeutische Diät überzuleiten.

Der individuelle therapeutische Ansatz wurde auch im Vortrag über pollenassoziierte Nahrungsmittelallergie von Anja Constien herausgestellt. Nach Sicherung der Diagnose sei es keinesfalls gerechtfertigt, alle bekannten Kreuzallergene zu meiden. Nachgewiesene Sensibilisierungen seien keine Gewähr dafür, dass die entsprechenden Nahrungsmittel auch tatsächlich zu klinisch relevanten Symp- tomen führen. Pauschale Lebensmittellisten sollten daher der Vergangenheit angehören! Die Ernährungstherapie bei pollenassoziierter Nahrungsmittelallergie hat vielmehr zum Ziel, eine individuelle Beratungsaussage zu erarbeiten.

Anhand von zwei Fallberichten setzte sich Mandy Ziegert, München, mit der Fragestellung auseinander, inwieweit Reaktionen in der doppelblinden plazebokontrollierten Nahrungsmittelprovokation (DBPCFC) vorhersehbar sind. Sie zeigte die Daten von zwei Säuglingen mit atopischer Dermatitis und Verdacht auf Kuhmilcheiweißallergie, die mittels DBPCFC provoziert wurden. Während das Kind mit positiven RAST-Befunden nicht reagierte, kam es bei dem Säugling ohne spezifisches IgE gegen Kuhmilcheiweiß zu einer deutlichen Reaktion nach einer Menge von 1,4 ml Kuhmilch. Sie unterstrich damit erneut, dass der Aufwand einer DBPCFC gerade bei Verdacht auf Grundnahrungsmittelallergie notwendig ist, um Auslöser sicher zu identifizieren, aber auch um unnötige Eliminationsdiäten zu vermeiden.

Kerstin Dobberstein, Berlin, gab einen Überblick über Ersatzprodukte bei Nahrungsmittelallergien in verschiedenen Lebensaltern. Während bei Säuglingen und Kleinkindern extensive Hydrolysatnahrungen oder Aminosäureformula im Vordergrund stehen, geht

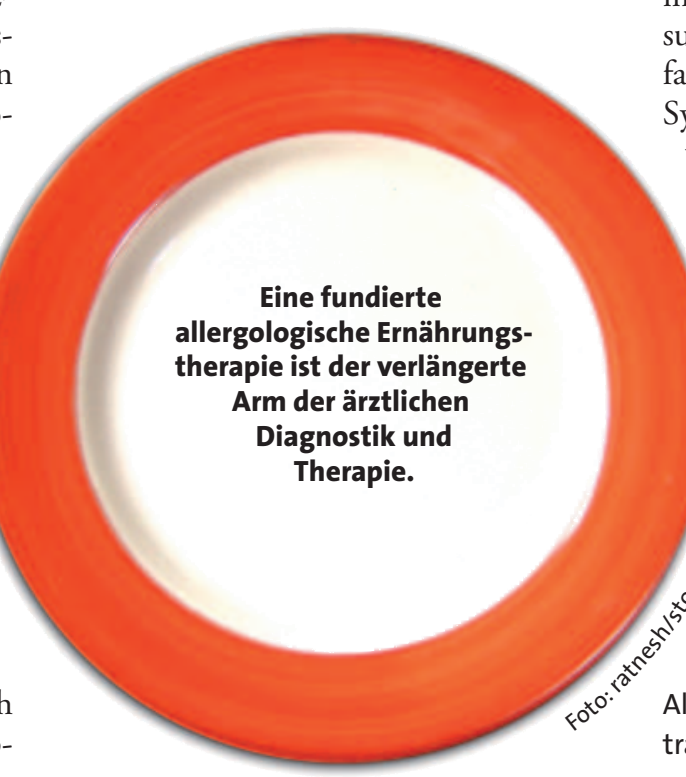

Arbeitskreis Diätetik stellt sich vor

Der 2005 in Aachen gegründete Arbeitskreis Diätetik in der Allergologie ist ein Zusammenschluss von Ernährungsfachkräften, die einen Beratungsschwerpunkt in der Allergologie aufweisen können. Eine Selbstverpflichtung zur regelmäßigen allergologischen Fortbildung und ein interner Austausch auf Fachebene stellen das hohe Qualitätsniveau der allergologischen Ernährungstherapie sicher. In Erfurt richtete der Arbeitskreis ein eigenes Symposium aus. Weitere Informationen bietet die Homepage www.ak-dida.de.

es mit zunehmendem Alter darum, adäquate Lebensmittel als Ersatz zu finden, die eine ausreichende Nährstoffdeckung sicherstellen und gleichzeitig schmackhaft wie kindgerecht sind.

Zum Abschluss des Symposiums berichtete Christiane Schäfer, Hamburg, von den Schwierigkeiten, den Betroffenen die Regelungen der aktuell gültigen Allergenkennzeichnung bei verpackter Ware zu vermitteln. Dabei geht es nicht nur um die bekannten Probleme unbeabsichtigter Einträge, fehlender Höchstmengenregelung oder der Beschränkung auf verpackte Ware. Anhand von beispielhaften Zutatenlisten demonstrierte sie, vor welchem Informationsdschungel die Betroffenen zum Teil stehen. So sind noch Listen nach alter Kennzeichnungsverordnung im Umlauf (z. B. Konserven, Tütensuppen), auch werden Begriffe oft nicht fachgerecht eingesetzt (z.B. Nüsse als Synonym für Schalenfrüchte) oder es werden Warnhinweise ohne therapeutische Konsequenz aufgedruckt (z.B. „Kann Spuren von Laktose enthalten"). Dr. Imke Reese, München

Kamp A. Histaminintoleranz aus diätetischer Sicht. Bunselmeyer B. Provokationsdiät bei chronischer Urtikaria. Constien A. Ernährungso therapie bei pollenassoziierten Nahrungsmittelallergien. Ziegert,

M. DBPCFC-Reaktionen vorausseh-

bar? Dobberstein K. Ersatzprodukte bei Nahrungsmittelallergie. Schäfer C. Allergenkennzeichnung 2008 - Praxistransfer für die tägliche Beratung 